\title{
Application programmes at the Scottish Centre for the Application of Plasma-based Accelerators (SCAPA)
}

\author{
S. M. Wiggins ${ }^{1}$, M. Boyd ${ }^{2}$, E. Brunetti ${ }^{1}$, N. M. H. Butler ${ }^{1}$, J. S. Feehan ${ }^{1}$, R. J. Gray ${ }^{1}$, B. Hidding ${ }^{1}$, \\ D. G. Ireland ${ }^{3}$, W. Li ${ }^{1}$, A. Maitrallain ${ }^{1}$, G. G. Manahan ${ }^{1}$, P. McKenna ${ }^{1}$, D. O’Donnell ${ }^{4}$, M. Scheck ${ }^{4}$, \\ M. Shahzad ${ }^{1}$, Z.-M. Sheng ${ }^{1}$, R. Spesyvtsev ${ }^{1}$, G. Vieux ${ }^{1}$, D. P. Watts ${ }^{5}$, G. H. Welsh ${ }^{1}$, R. Wilson ${ }^{1}$, \\ N. Zachariou ${ }^{5}$ and D. A. Jaroszynski ${ }^{1}$
}
${ }^{1}$ Scottish Universities Physics Alliance, Department of Physics, University of Strathclyde, Glasgow G4 0NG, UK and The Cockcroft Institute, Sci-Tech Daresbury, Keckwick Lane, Daresbury, Warrington WA4 4AD, UK
${ }^{2}$ Strathclyde Institute of Pharmacy and Biomedical Sciences, University of Strathclyde, Glasgow G4 0RE, UK
${ }^{3}$ Scottish Universities Physics Alliance, School of Physics and Astronomy, University of Glasgow, Glasgow G12 8QQ, UK
${ }^{4}$ Scottish Universities Physics Alliance, School of Engineering and Computing, University of the West of Scotland, Paisley PA1 2BE, UK
${ }^{5}$ Department of Physics, University of York, Heslington, York YO10 5DD, UK

\begin{abstract}
The Scottish Centre for the Application of Plasma-based Accelerators (SCAPA) is a research facility dedicated to providing high energy particle beams and high peak brightness radiation pulses for users across a wide range of scientific and engineering disciplines. A pair of Ti:sapphire femtosecond laser systems (40 TW peak power at $10 \mathrm{~Hz}$ pulse repetition rate and $350 \mathrm{TW}$ at $5 \mathrm{~Hz}$, respectively) are the drivers for a suite of laser-plasma accelerator beamlines housed across a series of radiation shielded areas. The petawatt-scale laser delivers $45 \mathrm{~W}$ of average power, which has established it as a world leader in its class. The University of Strathclyde has had an operational laser wakefield accelerator since 2007 as the centrepiece of the on-going Advanced Laser Plasma High-energy Accelerators towards X-rays (ALPHA-X) project. SCAPA, which is a multi-partner venture supported by the Scottish Universities Physics Alliance, continues the dedicated beamline approach pioneered by ALPHA-X and represents a significant expansion in the UK's experimental capability at the university level in laser-driven acceleration. The new centre supports seven dedicated radiation beamlines across three shielded bunkers that each nominally specialise in different aspects of fundamental laser-plasma interaction physics and radiation sources: $\mathrm{GeV}$-scale electron beams, $\mathrm{MeV}$ proton and ion beams, X-rays, gamma rays etc. Development of application programmes based on these sources cover a wide range of fields including nuclear physics, radiotherapy, space radiation reproduction, warm dense matter, high field physics and radioisotope generation.
\end{abstract}

Keywords: plasma accelerator, femtosecond lasers, wakefield, ion beams, Ti:sapphire, laser-plasma, applications, radiation sources

\section{INTRODUCTION}

Particle accelerators and radiation light sources based on radio-frequency (RF) or microwave driving fields are wellestablished research tools spanning a range of fundamental and applied scientific areas. ${ }^{1}$ The maximum sustainable accelerating fields are $\sim 100 \mathrm{MV} / \mathrm{m}$ (limited by cavity breakdown) and therefore large expensive infrastructures are required for high energy particle beamlines. X-ray synchrotron and free-electron laser (FEL) sources are based on mature RF technology, which has enabled large facility investments to be made, however, the small number of facilities and their high costs has led to over-subscription.

The recent development of high-power, ultrashort pulse lasers is leading to transformative accelerator technologies that have the potential to replace conventional accelerators and radiation sources for a wide range of applications. The high 
electric fields of intense laser pulses focussed onto gas, pre-formed plasma or solid targets can cause charge separation, which produces electrostatic fields with accelerating gradients that can be more than three orders of magnitude greater than in RF accelerator cavities. Such ultra-compact ( $\mu \mathrm{m}$ to $\mathrm{cm}$ scale) accelerating structures can form the basis of sources of high energy electrons, protons and ions, and high brightness X-ray and gamma ray pulses. ${ }^{2}$ Their unique characteristics, reduced requirement for large infrastructure and the development of more reliable lasers could eventually lead to widespread deployment across university, industry and medical environments with a hugely beneficial societal impact.

Worldwide investigation of laser-based acceleration is growing, largely due to the proliferation of petawatt-class high power Ti:sapphire laser systems ${ }^{3}$ and the variety of accelerating configurations that can be studied according to the desired output particle species and characteristics. Laser wakefield accelerators $\mathrm{s}^{4,5}$ are sources of femtosecond to attosecond duration pulses of relativistic electrons with $\mathrm{GeV}$ electron energies ${ }^{6}, \mathrm{X}$-rays and gamma rays ${ }^{7}$. Laser-solid interactions lead to $\mathrm{MeV}$ proton and ion beams, and secondary particles ${ }^{8,9}$ by target normal sheath acceleration, ${ }^{10}$ radiation pressure acceleration ${ }^{11}$ or break-out afterburner acceleration. ${ }^{12}$ Laser pulse repetition rates are currently a hurdle to be overcome for most future applications.

The recently established Scottish Centre for the Application of Plasma-based Accelerators (SCAPA), located at the University of Strathclyde, UK, is a centre devoted to the research, development and application of laser-driven accelerators. It is a multi-partner venture supported by the Scottish Universities Physics Alliance that will serve as a source provider for research groups from academia and industry. The workhorses in SCAPA are two commercial high power Ti:sapphire laser systems: one $5 \mathrm{~Hz}, 350 \mathrm{TW}$ system, and one $10 \mathrm{~Hz}, 40 \mathrm{TW}$ system. The $350 \mathrm{TW}$ laser is capable of delivering $45 \mathrm{~W}$ of average power (after compression), which represents the world leader in its class. In this paper, we outline the layout of SCAPA for studies across the whole gamut of particle species and accelerating regimes. Both laser systems will then be described. We conclude with a discussion on the perspectives of the development of laser-based accelerators and their applications.

\section{CENTRE LAYOUT}

The University of Strathclyde has had an operational laser wakefield accelerator since 2007 as the centrepiece of the on-going Advanced Laser Plasma High-energy Accelerators towards X-rays (ALPHA-X) project, which aims to develop compact coherent radiation sources, ${ }^{13}$ and SCAPA represents a significant expansion in the UK's experimental capability in developing laser-driven accelerators. The new centre comprises seven particle and radiation beamlines situated in three separate concrete shielded bunkers. These include proton and ion beamline areas that will support research in this area. ${ }^{14,15}$ Similar laser power has also recently been the driver for the generation of $\approx 8 \mathrm{GeV}$ electron beams from a laser wakefield accelerator. ${ }^{16} \mathrm{~A}$ way of working that has carried over from ALPHA-X into SCAPA is the concept of permanent accelerator beamlines that enable in-depth, high quality, systematic research programmes sustained over a long period of time. This idea underpins the design of the centre, which includes multiple beamlines, each of which specialise in different topics of laser-driven acceleration. Two driving laser systems allow simultaneous operation of two accelerator beamlines in different bunkers, while preparatory work is conducted in the third bunker.

SCAPA is laid out over two levels and the three bunkers form the bulk of the lower level. The laser labs are located on top of the bunkers on the second level. As shown in Figure 1, the 40 TW laser drives three beamlines (one in Bunker A and both in Bunker C), while the 350 TW laser drives four beamlines (two in Bunker A and both in Bunker B). The latter laser in Bunker B is first routed to a plasma mirror system for pulse contrast enhancement ${ }^{17}$ before delivery to the beamline. To maximise the working space within each bunker, concrete shielding doors that float on compressed air skates are utilised (instead of chicanes), as shown in Figure 2.

The bunker layout is suitable for handling the respective particle species generated in each beamline and, therefore, supports the research and application programmes. Bunker C houses the re-located ALPHA-X beamline and is devoted to laser wakefield acceleration of ultrashort electron bunches in the $100-300 \mathrm{MeV}$ range ${ }^{18,19}$ production of high brightness $\mathrm{X}$-ray betatron radiation ${ }^{7}$ and medical applications including medical imaging and very high energy electron beam radiotherapy. ${ }^{20}$ Bunker $\mathrm{A}$ is mainly devoted to laser wakefield acceleration studies and, with a working length of $23.5 \mathrm{~m}$, allows for development of coherent radiation sources such as a laser wakefield-driven X-ray free-electron laser. ${ }^{13,21,22}$ Other applications include replicating space radiation, ${ }^{23}$ Raman amplification and manipulation of intense laser pulses in plasma ${ }^{24}$ and X-ray detector development. ${ }^{25}$ Bunker B is configured for laser-solid target interactions towards the generation of 
proton and ion beams and secondary particles, and applications such as studies of warm dense matter, ${ }^{26}$ laser-driven fusion ${ }^{27}$ and radiography. ${ }^{28}$

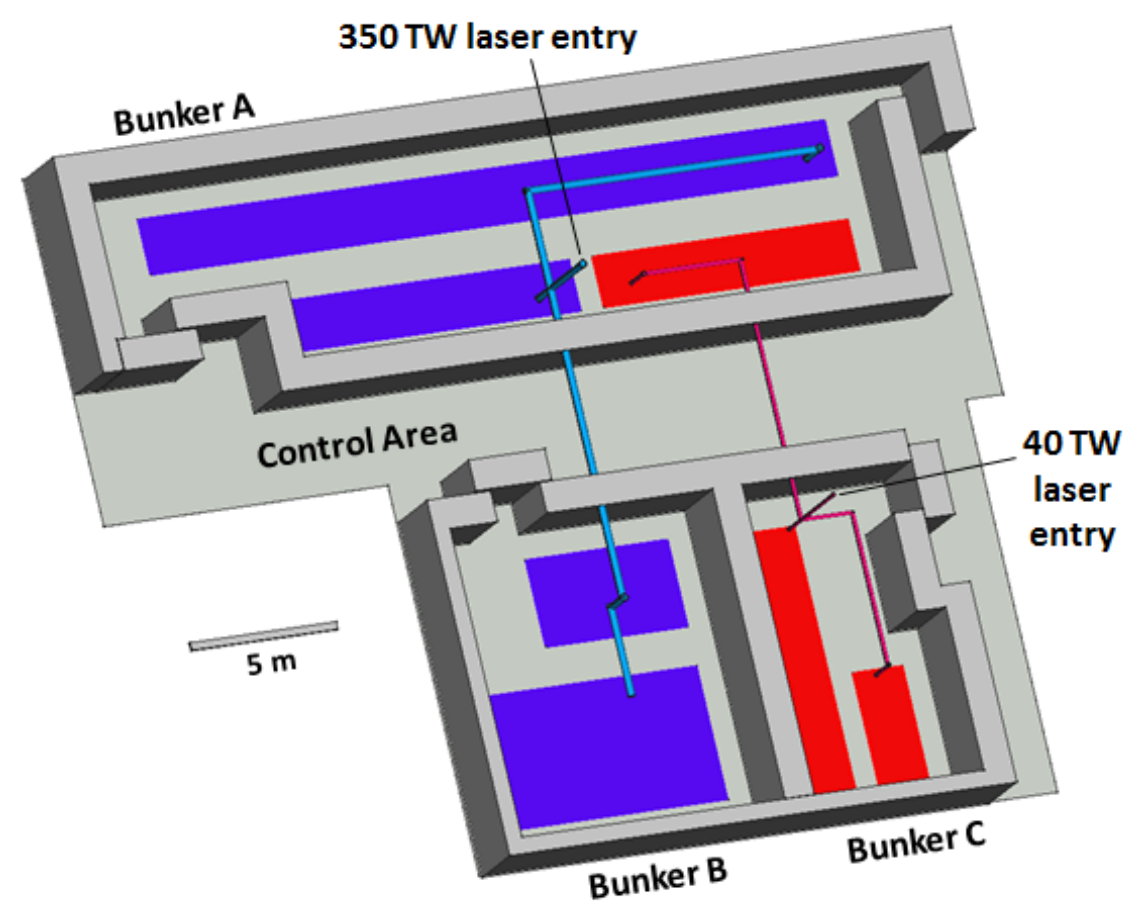

FIGURE 1. Layout of SCAPA radiation beamlines within the concrete bunkers. Red shaded regions are beamline areas provided by the $40 \mathrm{TW}$ laser. Blue shaded regions are beamline areas provided by the $350 \mathrm{TW}$ laser.

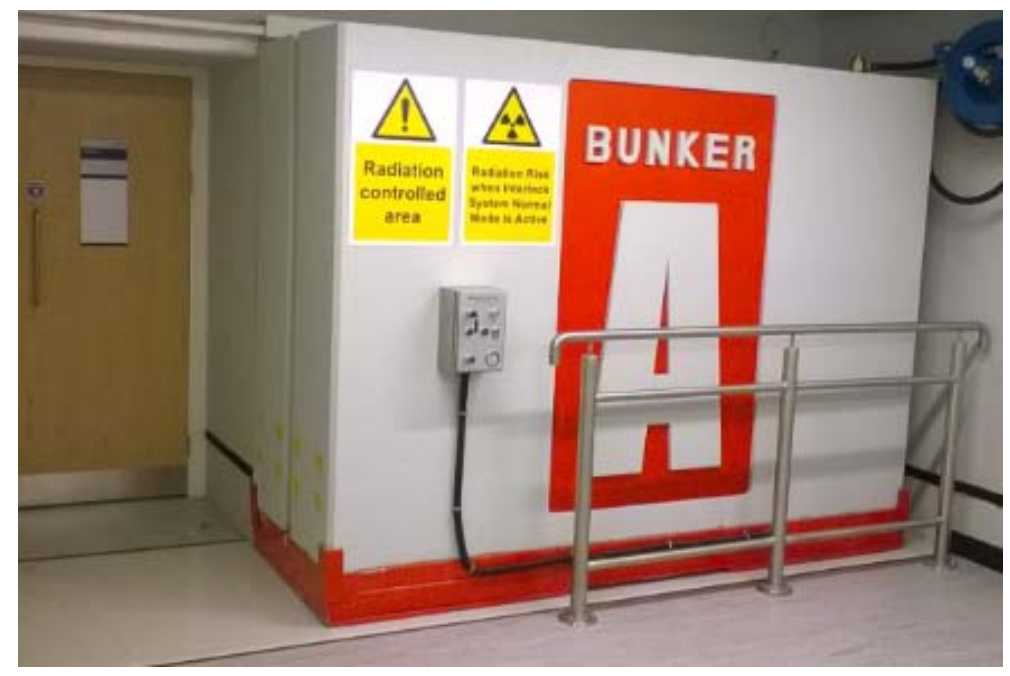

FIGURE 2. One of the pneumatically driven concrete shielding doors.

SCAPA also includes a number of auxiliary rooms (fume cupboards, biological safety cabinets, image plate reader, radioactive sealed sources, etc.) for user preparations and post-analysis. A third laser lab features a $1 \mathrm{kHz}$ repetition rate Ti:sapphire laser system ( $3 \mathrm{~mJ}$ per pulse) that is used for plasma diagnostic development, accelerator off-line testing and femtosecond laser micromachining of wakefield capillary waveguides ${ }^{13,29}$ and particle and photon beam filters.

Laser pulses are transported under vacuum through the laser lab floors and bunker ceilings to their beamline destinations. Distances from the laser vacuum compressor output to the destinations are up to $25 \mathrm{~m}$, therefore, in order to 
minimise pointing fluctuations in the laser beam at target, SCAPA is vibrationally isolated using damping systems that isolate the bunkers, control area and TW laser labs from external vibrations. The bunkers, consisting of nearly 2,000 tons of concrete on the ground floor, are naturally stable, however, specific measures were required to provide high stability in the laser labs situated on top of the bunkers. This was complicated by the fact that SCAPA comprises one partrefurbishment of an existing building and another part that is a new building, such that load-bearing support columns in the western half of SCAPA had to be maintained. The result is that the $40 \mathrm{TW}$ laser lab (on the pre-existing building side) satisfies the generic vibration ${ }^{30}$ criterion VC-C $(12.5 \mu \mathrm{m} / \mathrm{s}$ rms speed value) while the $350 \mathrm{TW}$ laser lab (on the new build side) meets the more stringent VC-E $(3.1 \mu \mathrm{m} / \mathrm{s})$ standard.

\section{40 TW LASER SYSTEM}

This laser has been the mainstay of the ALPHA-X project since its inception in 2002 and has undergone a number of upgrades since then. It is presently composed of a mixture of amplifier sub-systems from both Thales Optronique and Amplitude Technologies (see Table 1), with specifications given in Table 2. A photograph of the laser lab is shown in Figure 3.

\begin{tabular}{|c|c|c|}
\hline \multicolumn{2}{|c|}{ Sub-system } & Pulse Energy \\
\hline Oscillator & Femtolasers: Femtosource Synergy pumped by & $9 \mathrm{~nJ}$ \\
\hline Booster & Ampl. Tech.: pumped by CFR Ultra & $40 \mu \mathrm{J}$ \\
\hline Regenerative Amplifier & Ampl. Tech.: pumped by CFR Ultra \& CFR 200 & $200 \mu \mathrm{J}$ \\
\hline Multi-pass Amplifier 1 & Ampl. Tech.: 4-pass pumped by CFR 200 & $30 \mathrm{~mJ}$ \\
\hline Multi-pass Amplifier 2 & Thales: 3-pass pumped by 2 $\times$ Saga & $300 \mathrm{~mJ}$ \\
\hline Multi-pass Amplifier 3 & Thales: 4-pass pumped by 2 $\times$ Saga \& 2 $\times$ Saga HP & $2 \mathrm{~J}$ \\
\hline
\end{tabular}

TABLE 1. Major sub-systems of the 40 TW laser system.

\begin{tabular}{|l|c|}
\hline Pulse repetition rate & $10 \mathrm{~Hz}$ \\
\hline Central wavelength & $800 \mathrm{~nm}$ \\
\hline Energy per pulse (after compression) & $1.4 \mathrm{~J}$ \\
\hline Full-width at half-maximum pulse duration & $35 \mathrm{fs}$ \\
\hline Probe beam energy per pulse (uncompressed) & $30 \mathrm{~mJ}$ \\
\hline
\end{tabular}

TABLE 2. Specifications of the 40 TW laser system.

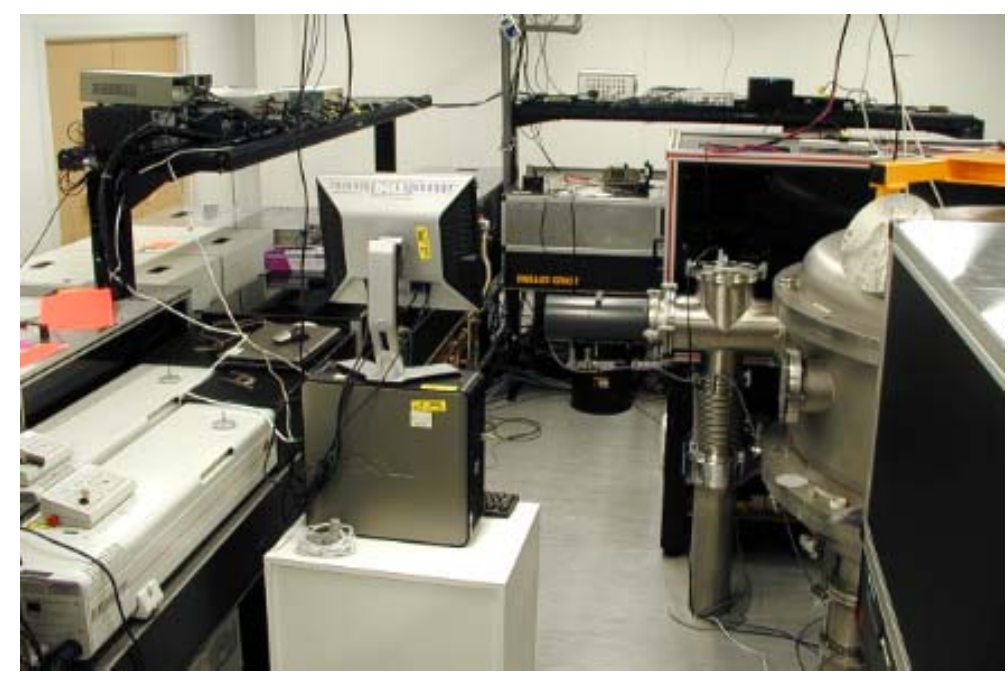

FIGURE 3. 40 TW laser system that delivers the beam through the floor (lower right) to Bunker C below. 


\section{350 TW LASER SYSTEM}

Custom-built by Thales Optronique, this laser was commissioned on-site in early 2017 as the world's first petawattclass laser operating at $5 \mathrm{~Hz}$. Specifications and sub-systems are shown in Tables 3 and 4, respectively. It is situated in a class 1000 ISO 6 cleanroom with all major power supply units located in an adjacent room to minimise heat loading in the cleanroom. A photograph of the cleanroom laser lab is shown in Figure 4. High contrast ratio is achieved with the crosspolarised wave generation filter system ${ }^{31}$ as patented by Thales and Laboratoire d'Optique Appliquée. ${ }^{32}$

\begin{tabular}{|l|c|}
\hline Pulse repetition rate & $5 \mathrm{~Hz}$ \\
\hline Central wavelength & $800 \mathrm{~nm}$ \\
\hline Energy per pulse (after compression) & $8.75 \mathrm{~J}$ \\
\hline Full-width at half-maximum pulse duration & $25 \mathrm{fs}$ \\
\hline & $10^{10}: 1 @ 100 \mathrm{ps}$ \\
Contrast ratio & $10^{8}: 1 @ 30 \mathrm{ps}$ \\
& $10^{4}: 1 @ 2 \mathrm{ps}$ \\
\hline Strehl ratio & $\geq 0.85$ \\
\hline Probe beam energy per pulse (uncompressed) & $30 \mathrm{~mJ}$ \\
\hline
\end{tabular}

TABLE 3. Specifications of the 350 TW laser system.

\begin{tabular}{|c|c|c|}
\hline \multicolumn{2}{|c|}{ Sub-system } & Pulse Energy \\
\hline Oscillator & Coherent: Vitara S pumped by Coherent Verdi G & $8 \mathrm{~nJ}$ \\
\hline Regenerative Amplifier & pumped by Jade 2 & $600 \mu \mathrm{J}$ \\
\hline Booster & pumped by Jade 2 & $60 \mu \mathrm{J}$ \\
\hline Pre-Amplifier 1 & 4-pass pumped by 3 $\times$ Saga HP & $35 \mathrm{~mJ}$ \\
\hline Multi-pass Amplifier 1 & 3-pass pumped by 3 $\times$ Saga HP & $1.4 \mathrm{~J}$ \\
\hline Multi-pass Amplifier 2 & 3-pass pumped by 2 $\times$ GAIA HP & $14 \mathrm{~J}$ \\
\hline
\end{tabular}

TABLE 4. Major sub-systems of the 350 TW laser system.

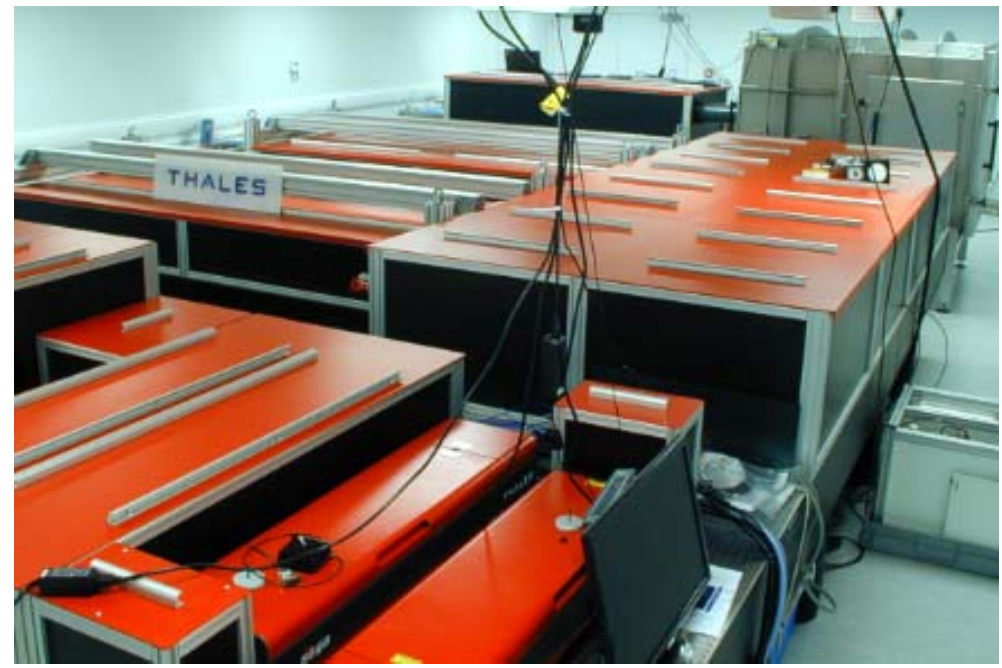

FIGURE 4. $350 \mathrm{TW}$ laser system that delivers the beam through the floor (beyond the grey vacuum compressor chamber upper right) to Bunker A below. 


\section{APPLICATION PROGRAMMES}

SCAPA is open to user engagement across all areas of research, including development of primary or secondary sources, proof-of-principle demonstrations or application of the sources. Source development is a major aspect of the research because laser-plasma acceleration (using underdense or overdense targets) is still a maturing field in terms of exploring, controlling and stabilising parameters such as source brightness, pointing, spectral range and pulse duration. SCAPA is an important test centre for demonstrating and applying new concepts. This is pertinent for applications requiring very high pulse repetition rates $(\mathrm{kHz}$ to $100 \mathrm{~s}$ of $\mathrm{kHz})$, which will require future laser technology upgrades. Other applications will require mobile laser-plasma accelerators in order to take the source to the sample.

SCAPA is an ideal testing platform for new ideas, such as controlled electron injection ${ }^{19}$ for the acceleration of unique electron bunches and multi-GeV electron beams. It has been shown in 1-D and 2-D simulations ${ }^{33}$ and 3-D simulations ${ }^{34}$ that attosecond electron bunches are injected at the density transition from the up-ramp to the uniform region. Methods of controlling both the duration and the charge of injected bunches using a tailored down-ramp section (a short plasma density bump) of the plasma have been developed, where the flat plasma region is kept below the density threshold for injection, leading to controlled injection of attosecond bunches. ${ }^{35}$ In all these cases, the phase velocity changes from superluminal (electron velocity below threshold for injection) to subluminal (electron velocity above threshold for injection) in the density transition region, leading to injection. Another scheme is ionisation-controlled electron injection in the so-called self-truncation regime ${ }^{36}$ where the laser spot size is not matched to the plasma density and electron injection only occurs over a short distance, producing electron bunches with narrow energy spreads. ${ }^{19}$ Laser propagation through tailored preionised plasma channels is also under investigation. ${ }^{37}$ With the SCAPA 350 TW laser, multi-GeV quasi-monoenergetic ultra-short electron bunches with exceptionally high peak currents are expected using these schemes.

With high energy electron bunches produced stably via the schemes as mentioned above, ultrashort coherent radiation can be produced. The longest beamline in SCAPA (visible in Bunker A towards the top of Figure 1) facilitates exploration of the LWFA as a driver for an FEL ${ }^{13,21,22,38}$ which has been an ambition in the community ever since the first demonstration of spontaneous undulator radiation with such a driver in $2008 .^{39,40}$ Electron bunches of energy up to 2 GeV in SCAPA will generate undulator radiation with wavelengths down to $0.5 \mathrm{~nm}(2.5 \mathrm{keV})$, i.e., encompassing the $2.3-4.4 \mathrm{~nm}$ "water window". To achieve FEL self-amplified spontaneous emission, a low 6D emittance beam and high quality beam transport system is required. The ultimate goal is to demonstrate a cost-effective alternative to conventional X-ray FELs, which require large investments, occupy kilometres of infrastructure and have high running costs.

One step beyond replacement of the conventional accelerator with a plasma accelerator is the replacement of the conventional undulator with a plasma undulator such as in the ion channel laser. ${ }^{41,42}$ It has been shown theoretically and numerically that attosecond electron bunches can directly lead to attosecond XUV radiation in the 10-100 eV range via coherent synchrotron radiation inside the bubble. ${ }^{43}$ The energy of such an attosecond pulse can reach the mJ level, several orders of magnitude higher than those produced via high harmonic generation in gas. Moreover, betatron radiation intensity and critical photon energy can be enhanced when accelerated electrons interact with the laser pulse resulting in betatron resonance. ${ }^{7,44}$ These novel radiation sources are of interest for advancing photon-based applications.

The inherently short pulses and large peak currents of electron beams produced by an LWFA stage will also be exploited to realise electron beam-driven plasma wakefield accelerator (PWFA). ${ }^{45}$ This hybrid approach will harness the distinct features of the PWFA, such as dephasing-free operation, long acceleration distances and novel injection schemes such as the plasma photocathode, ${ }^{46}$ which promises unprecedented electron beam brightness. ${ }^{47}$ Such electron beams may have transformative impact on applications, such as realising $5^{\text {th }}$ generation light sources. ${ }^{48,49}$

The extreme beams that are realisable by plasma accelerators and, in particular, by novel injection schemes such as down-ramp injection ${ }^{35}$ and the plasma photocathode ${ }^{46}$ also require novel diagnostics and measurement techniques. In this application theme, the plasma response to the interaction of electron beams and laser pulses with plasma will be exploited as a highly sensitive 'magnifying glass' to retrieve multi-dimensional fingerprints of the interaction. For example, the transient spatio-temporal overlap between electron beam and laser beams can yield hot spots manifested by a sudden burst of additional plasma due to transient tunnelling ionisation. Such effects have been observed at the SLAC National Accelerator laboratory FACET facility ${ }^{50}$ and may constitute a novel generation of plasma diagnostics, suitable for measuring intense beam form factors, emittance and brightness directly at the interaction point without need for any mechanical equipment close to the interaction region. These laser-gated plasma diagnostics will be co-developed with 
industry for use at SCAPA and on other accelerator systems (both conventional and plasma-based). Methods of measuring ultrashort bunches using coherent transition radiation will also be used to measure the duration of attosecond bunches. ${ }^{19}$

SCAPA is also an ideal environment for the development of plasma-based optics relevant to next-generation high intensity laser systems. The quest for ever higher laser intensity to explore new regimes in high field physics ${ }^{51}$ is driving research into plasma amplification media and nonlinear optical components. This includes stimulated Raman backscattering in plasma ${ }^{24,52}$ acting as a direct amplifier of ultrashort laser pulses that has the potential to underpin realisation of compact exawatt laser systems. A wide variety of other plasma phenomena, such as plasma photonic crystals, ${ }^{53}$ induced transparency ${ }^{14}$ and high harmonic generation ${ }^{54}$ are open for investigation as well.

The research programme in bunker B will focus on the development and application of particle and radiation sources generated from intense laser interactions with dense plasma (typically thin foils, foams and potentially high density gas jets). Standard thin foils will be used in the first experiments to characterise the properties of protons beams produced via the target normal sheath acceleration mechanism. ${ }^{10}$ Double-plasma mirror beam-conditioning will enable ultra-high contrast laser pulses to be delivered ${ }^{17}$ and thus experimentation with ultrathin target foils. This will enable investigation of promising ion acceleration schemes involving thin foils expanding to near-critical densities, including radiation pressuredriven acceleration and hybrid schemes involving transparency-enhanced acceleration..$^{55} \mathrm{~A}$ dedicated beamline, which can be developed and optimised over time will enable the fundamental relativistic laser-plasma physics that underpins the development of these acceleration schemes to be investigated. This includes the role of relativistic-induced transparency, ${ }^{14}$ which can open up new degrees of control on laser-driven ion acceleration. ${ }^{15}$ The transition between acceleration mechanisms, enabled by the use of ultrathin foils and control of the laser pulse parameters, will also be investigated. The high repetition rate capability of the 350 TW laser at SCAPA will enable dense plasma studies at the forefront of the field to be conducted with unprecedented detail and statistical significance. This will support a greater understanding of the underpinning physics of novel laser-driven particle and radiation sources in dense plasma.

A wide part of the electromagnetic spectrum is covered in SCAPA by available photon sources, either direct from the plasma or as a secondary source, from high energy gamma rays generated via bremsstrahlung radiation ${ }^{56,57}$ to $\mathrm{THz}$ radiation, ${ }^{58,59}$ the latter being produced with energies as high as tens of millijoules via irradiation of metal foils. ${ }^{60}$ For imaging purposes, the small plasma source size leads to excellent image resolution that may be further enhanced by coherent effects, such as in betatron radiation phase-contrast imaging. ${ }^{7,61}$ Gamma rays can also be produced direct from the plasma as in the case of LWFA resonant betatron radiation ${ }^{7}$ or, in dense targets, via the generation of multi-megaAmpere currents of relativistic electrons,$^{57}$ the latter process also driving the generation of mega-Gauss magnetic fields. The acceleration of light ions enables beamed neutron emission via fusion and transmutation reactions in a secondary target position downstream ${ }^{62}$, which enable a great variety of potential imaging applications. Imaging of dense material, for example, for security application, radiometric assaying or industrial radiography can be achieved with gamma rays or neutrons. ${ }^{28,57}$ The ultrashort pulse nature of the radiation will look to be exploited in remote sensing applications.

SCAPA has the facilities to conduct on-going research into the use of hadron ${ }^{63}$ and electron ${ }^{64}$ beams as potential treatment modalities for radiotherapy. Proton beam therapy, that exploits the advantageous dose deposition profile of protons/ions (the characteristic Bragg peak) generally requires beam energy in the range $200-250 \mathrm{MeV}$ which is beyond the current experimental limits of laser-plasma acceleration. However, energy of around $70 \mathrm{MeV}$ is sufficient for some ocular tumours ${ }^{63}$ and this is now on the horizon of accelerators driven by a laser of the scale of the SCAPA 350 TW system. Very high energy electrons, on the other hand, utilising electrons of $100 \mathrm{MeV}$ or more ${ }^{64,65}$ are promising sources for deepseated tumours because their dose deposition profile is superior to conventional X-rays and potentially comparable with protons, depending on the particular geometry and dose delivery plan. For both hadron and electron beams, there is still much to understand with respect to the fundamental interaction between such ultrashort particle beams delivering doses at ultrahigh rates and biological tissue. ${ }^{66}$

Laser-plasma accelerator radiotherapy studies can readily deliver the absorbed dose for patient treatment. ${ }^{20,66}$ However, clearly the laboratory environment is not a clinical setting for patient trials. This illustrates the proof-of-principle concept of the centre. The same is true for production of medical radioisotopes, where the low cost of a compact laser-driven system could enable local production of short-lived isotopes on a hospital campus. The entire periodic table is open to investigation, ranging from light isotopes such as $\mathrm{C}-11$ and $\mathrm{F}-18$ for imaging ${ }^{67}$ to Ac-225 and $\mathrm{Pb}-212$ for targeted alpha therapy. ${ }^{68}$ Currently, relatively low activity levels $(\sim \mathrm{kBq})$ can be generated by electron beam-driven photonuclear ${ }^{69}$ or 
direct proton beam-driven ${ }^{70}$ reactions and future up-scaling of beam charge and pulse repetition rate may allow meaningful activity levels ( $\sim \mathrm{MBq}-\mathrm{GBq})$ of a radioisotope to be produced. It has been shown that the LWFA can produce bunches with $\mathrm{nC}$ charge levels in divergence beams of electrons with $\mathrm{MeV}$ energy ${ }^{71}$ and exploiting this high charge for application is an enticing prospect. High repetition rate accelerators rely on laser technology advancements in fibres ${ }^{72,73}$ and/or solid state devices $^{74,75}$ to overcome the relatively high running costs of equivalent flashlamp-driven laser systems.

Further nuclear applications include detector development and isomer excitation. Laser-plasma accelerator beams are highly suited to the development of many varieties of advanced particle detectors. These include silicon and germanium solid state detectors, such as silicon photomultipliers, high purity germanium detectors and silicon pixel detectors. ${ }^{25}$ Also, $\mathrm{keV}$ photon beams can be used to develop and test radiation-hard diamond detectors, while electron beams can be used to test electromagnetic calorimeters ${ }^{76}$ - the same technology that is used in positron emission tomography (PET) scanners and combined PET/magnetic resonance imaging scanners. ${ }^{77}$ In large-scale nuclear or particle physics experiments, Cherenkov and transition radiation detectors have become key components and enhancements of these systems require very precise timing and space point reconstruction of the photons generated inside the fused silica radiator; ${ }^{78}$ ultrashort beams at SCAPA can enable such studies. Novel detection systems can be directly applied in investigation of the structural evolution of nuclear core properties on atomic timescales at SCAPA, as well as being used at other facilities. ${ }^{79}$ Isomeric low-lying nuclear levels in stable isotopes and their response to ultrashort pulses can be studied with high peak brilliance photon sources ${ }^{80}$ Detector development work in this field includes obtaining sub-picosecond time resolution and extremely good signal-to-noise ratio, since the cross-sections of concern are much smaller than those for optical transitions.

The final application area outlined in this paper concerns radiation in space, which is a significant hazard for satellites and crewed spaceflight due to the missing protection of the Earth's magnetic field. This radiation can consist of electrons, protons and ions, with a broadband energy distribution, typically exponential or power-law shaped. While conventional radiation sources used for space radiation hardness assurance (RHA) ${ }^{81}$ produce unnatural, monoenergetic beams, the inherent capability of plasma accelerators to produce broadband beams offers a path to complementary space RHA with a very high level of realism by reproducing space radiation in the laboratory. ${ }^{23}$ This approach will be further developed in collaboration with space agencies, industry and other stakeholders. In addition to electronics testing, this research is also relevant to space radiobiology studies and other RHA areas, for example, in the nuclear arena.

\section{CONCLUSIONS}

Laser-plasma accelerators have great potential for societal impact and SCAPA is one of a growing number of research facilities worldwide devoted to exploring this potential and bringing it to fruition. With its multiple accelerator beamline approach and flexible user spaces, SCAPA aims to support a wide array of academic and industrial applications of this novel technology. High quality laboratory surroundings with excellent environmental stability (temperature, relative humidity, vibrations) provide an excellent environment for high impact laser-plasma accelerator research to be conducted at SCAPA.

\section{ACKNOWLEDGEMENTS}

We acknowledge support of the U.K. EPSRC (grant no. EP/K011952/1, EP/J018171/1, EP/N028694/1, EP/J500094/1, EP/P020607/1, EP/R006202/1), the Scottish Funding Council, the European Union's Horizon 2020 research and innovation programme under grant agreement no. 654148 Laserlab-Europe and grant agreement no. 653782 EuPRAXIA and also the the European Union's Extreme Light Infrastructure (ELI) Project.

\section{REFERENCES}

[1] Wiedemann, H., [Particle Accelerator Physics], Fourth Edition, Springer, New York (2015).

[2] Ledingham, K. W. D. and Galster, W., "Laser-driven particle and photon beams and some applications", New J. Phys. 12,045005 (2010).

[3] Danson, C., Hillier, D., Happs, N. and Neely, D., "Petawatt class lasers worldwide", High Power Laser Sci. Eng. 3, e3 (2015).

[4] Tajima, T. and Dawson, J. M., "Laser electron accelerator", Phys. Rev. Lett. 43, 267 (1979).

[5] Hooker, S. M., "Developments in laser-driven plasma accelerators", Nature Photon. 7, 775 (2013).

[6] Leemans, W. P. et al., "GeV electron beams from a centimetre-scale accelerator", Nature Phys. 2, 696 (2006).

[7] Cipiccia, S. et al., "Gamma-rays from harmonically resonant betatron oscillations in a plasma wake," Nature Phys. 7, 867 (2011). 
[8] Daido, H., Nishiuchi, M. and Pirozhkov, A. S., "Review of laser-driven ion sources and their applications", Rep. Prog. Phys. 75, 056401 (2012).

[9] Macchi, A., Borghesi, M. and Passoni, M., “Ion acceleration by superintense laser-plasma interaction”, Rev. Mod. Phys. 85, 751 (2013).

[10] Wilks, S. C. et al., "Energetic proton generation in ultra-intense laser-solid interactions", Phys. Plasmas 8, 542 (2001).

[11] Esirkepov, T. et al., "Highly efficient relativistic-ion generation in the laser-piston regime", Phys. Rev. Lett. 92, 175003 (2004).

[12] Yin L., Albright, B. J., Hegelich, B. M. and Fernández, J. C., "GeV laser ion acceleration from ultrathin targets: the laser break-out afterburner", Laser Part. Beams 24, 291 (2006).

[13] Jaroszynski, D. A. et al., "Radiation sources based on laser-plasma interactions", Phil. Trans. R. Soc. A 364,689 (2006).

[14] Gonzalez-Izquierdo, B. et al., "Optically controlled dense current structures driven by relativistic plasma apertureinduced diffraction", Nature Phys. 12, 505 (2016).

[15] Gonzalez-Izquierdo, B. et al., "Towards optical polarization control of laser-driven proton acceleration in foils undergoing relativistic transparency", Nature Commun. 7, 12891 (2016).

[16] Gonsalves, A. J. et al., "Petawatt Laser Guiding and Electron Beam Acceleration to 8 GeV in a Laser-Heated Capillary Discharge Waveguide", Phys. Rev. Lett. 122, 084801 (2019).

[17] Doumy, G. et al., "Complete characterization of a plasma mirror for the production of high-contrast ultraintense laser pulses", Phys. Rev. E 69, 026402 (2004).

[18] Brunetti, E. et al., "Low emittance, high brilliance relativistic electron beams from a laser-plasma accelerator", Phys. Rev. Lett. 105, 215007 (2010).

[19] Islam, M. R. et al., "Near-threshold electron injection in the laser-plasma wakefield accelerator leading to femtosecond bunches", New J. Phys. 17, 093033 (2015).

[20] Subiel, A. et al., "Dosimetry of very high energy electrons (VHEE) for radiotherapy applications", Phys. Med. Biol. 59, 5811 (2014).

[21] Jaroszynski, D. A. and Vieux, G., "Coherent radiation sources based on laser plasma accelerators", AIP Conf. Proc. 647, 902 (2002).

[22] Anania, M. P. et al., "An ultrashort pulse ultra-violet radiation undulator source driven by a laser plasma wakefield accelerator”, Appl. Phys. Lett. 105, 264102 (2014).

[23] Hidding, B. et al., "Laser-plasma-based space radiation reproduction and testing in the laboratory", Sci. Rep. 7, 42354 (2017).

[24] Yang, X. et al., "Chirped pulse Raman amplification in warm plasma: towards controlling saturation”, Sci. Rep. 5, 13333 (2015).

[25] Cipiccia, S. et al., "Compton scattering for spectroscopic detection of ultra-fast, high flux, broad energy range Xrays", Rev. Sci. Instrum. 84, 113302 (2013).

[26] Vaisseau, X. et al., "Enhanced relativistic-electron-beam energy loss in warm dense aluminum”, Phys. Rev. Lett. 114, 095004 (2015).

[27] Alejo, A. et al., "Numerical study of neutron beam divergence in a beam-fusion scenario employing laser driven ions", Nucl. Instrum. Methods Phys. Res., Sect. A 829, 176 (2016).

[28] Brenner, C. M. et al., "Laser-driven x-ray and neutron source development for industrial applications of plasma accelerators", Plasma Phys. Control. Fusion 58, 014039 (2016).

[29] Wiggins, S. M. et al., "Note: femtosecond laser micromachining of straight and linearly tapered capillary discharge waveguides", Rev. Sci. Instrum. 82, 096104 (2011).

[30] Segerink, F. B., Korterik, J. P. and Offerhaus, H. L., "Vibration transfers to measure the performance of vibration isolated platforms on site using background noise excitation", Rev. Sci. Instrum. 82, 065111 (2011).

[31] Jullien, A. et al., "Highly efficient temporal cleaner for femtosecond pulses based on cross-polarized wave generation in a dual crystal scheme", Appl. Phys. B 84, 409 (2006).

[32] Albert, O. et al., "Filtre non linéaire d'impulsions femtosecondes à contraste élevé", patent: (French, European and US) 11 2004. Publication number: 2878657 (int Cl: H01 S 3/10).

[33] Li, F.-Y. et al., "Dense attosecond electron sheets from laser wakefields using an up-ramp density transition", Phys. Rev. Lett. 110, 135002 (2013).

[34] Weikum, M, Li, F.-Y., Assmann, R. W., Sheng, Z. M. and Jaroszynski, D. A., "Generation of attosecond electron bunches in a laser-plasma accelerator using a plasma density up ramp", Nucl. Instrum. Methods Phys. Res., Sect. A 829, 33 (2016). 
[35] Tooley, M. P. et al., "Towards attosecond high-energy electron bunches: controlling self-injection in laser-wakefield accelerators through plasma-density modulation”, Phys. Rev. Lett. 119, 044801 (2017).

[36] Zeng, M. et al., "Self-truncated ionization injection and consequent monoenergetic electron bunches in laser wakefield acceleration", Phys. Plasmas 21, 030701 (2014).

[37] Abuazoum, S. et al., "Linearly tapered discharge capillary waveguides as a medium for a laser plasma wakefield accelerator", Appl. Phys. Lett. 100, 014106 (2012).

[38] Wiggins, S. M. et al., "Undulator radiation driven by laser-wakefield accelerator electron beams", Proc. SPIE 9509, 95090K (2015).

[39] Schlenvoigt, H.-P. et al., "A compact synchrotron radiation source driven by a laser plasma wakefield accelerator", Nature Phys. 4, 130 (2008).

[40] Gallacher, J. G. et al., “A method of determining narrow energy spread electron beams from a laser plasma wakefield accelerator using undulator radiation”, Phys. Plasmas 16, 093102 (2009).

[41] Whittum, D. H., Sessler, A. M. and Dawson, J. M., "Ion-channel laser”, Phys. Rev. Lett. 64, 2511 (1990).

[42] Ersfeld, B. et al., "The ion channel free-electron laser with varying betatron amplitude", New J. Phys. 16, 093025 (2014).

[43] Li, F.-Y. et al., "Radially polarized, half-cycle, attosecond pulses from laser wakefields through coherent synchrotron radiation", Phys. Rev. E 90, 043104 (2014).

[44] Huang, K. et al., "Resonantly enhanced betatron hard X-rays from ionization injected electrons in a laser plasma accelerator", Sci. Rep. 6, 27633 (2016).

[45] Hidding, B. et al., "Monoenergetic energy doubling in a hybrid laser-plasma wakefield accelerator", Phys. Rev. Lett. 104, 195002 (2010).

[46] Hidding, B. et al., "Ultracold electron bunch generation via plasma photocathode emission and acceleration in a beamdriven plasma blowout", Phys. Rev. Lett. 108, 035001 (2012).

[47] Manahan, G. G. et al., "Single-stage plasma-based correlated energy spread compensation for ultrahigh 6D brightness electron beams", Nature Commun. 8, 15705 (2017).

[48] Hidding, B. et al., "Ultrahigh brightness bunches from hybrid plasma accelerators as drivers of 5th generation light sources", J. Phys. B: At. Mol. Opt. Phys. 47, 234010 (2014).

[49] Gilljohann, M. F. et al., "Direct observation of plasma waves and dynamics induced by laser-accelerated electron beams", Phys. Rev. X. In press.

[50] Joshi, C. et al., "Plasma wakefield acceleration experiments at FACET II", Plasma Phys. Control. Fusion 60, 034001 (2018).

[51] Di Piazza, A., Müller, C., Hatsagortsyan, K. Z. and Keitel, C. H., "Extremely high-intensity laser interactions with fundamental quantum systems", Rev. Mod. Phys. 84, 1177 (2012).

[52] Vieux, G. et al., "An ultra-high gain and efficient amplifier based on Raman amplification in plasma", Sci. Rep. 7, 2399 (2017).

[53] Lehmann, G and Spatschek, K. H., "Transient plasma photonic crystals for high-power lasers", Phys. Rev. Lett. 116, 225002 (2016).

[54] Brunetti, E. Issac, R. and Jaroszynski, D. A., "Quantum path contribution to high-order harmonic spectra" Phys. Rev. A 77, 023422 (2008).

[55] Higginson, A. et al., "Near-100 MeV protons via a laser-driven transparency-enhanced hybrid acceleration scheme", Nature Commun. 9, 724 (2018).

[56] Cipiccia, S. et al., "A tuneable ultra-compact bright gamma-ray source based on bremsstrahlung radiation from laserplasma accelerated electrons", J. Appl. Phys. 111, 063302 (2012).

[57] Armstrong, C. D. et al., "Bremsstrahlung emission from high power laser interactions with constrained targets for industrial radiography", High Power Laser Sci. Eng. In press.

[58] Yang, X., Brunetti, E. and Jaroszynski, D. A., "High-energy coherent terahertz radiation emitted by wide-angle electron beams from a laser-wakefield accelerator", New J. Phys. 20, 043046 (2018).

[59] Zheng, Z. et al., "Manipulation of polarizations for broadband terahertz waves emitted from laser plasma filaments", Nature Photon. 12, 554 (2018).

[60] Liao, G. Q. et al., "Multi-millijoule coherent terahertz bursts from picosecond laser-irradiated metal foils", Proc. Natl. Acad. Sci. USA. Published online ahead of print, https://doi.org/10.1073/pnas.1815256116 (2019).

[61] Cipiccia, S. et al., "Inclusion of coherence in Monte Carlo models for simulation of x-ray phase contrast imaging", Opt. Express 22, 23480 (2014).

[62] Kar, S., "Beamed neutron emission driven by laser accelerated light ions", New J. Phys. 18, 10 (2016). 
[63] Ledingham, K. W. D., Bolton, P. R., Shikazono, N. and Charlie Ma, C.-M., "Towards laser driven hadron cancer radiotherapy: A review of progress", Appl. Sci. 4, 402 (2014).

[64] DesRosiers, C., Moskvin, V., Bielajew, A. F. and Papiez, L., “150-250 MeV electron beams in radiation therapy”, Phys. Med. Biol. 45, 1781 (2000).

[65] Kokurewicz, K. et al., "Laser-plasma generated very high energy electrons (VHEEs) in radiotherapy", Proc. SPIE 10239, 102390C (2017).

[66] Hanton, F. et al., "DNA DSB repair dynamics following irradiation with laser-driven protons at ultra-high dose rates", Sci. Rep. In press.

[67] Pimlott, S. L., "Radioisotopes for medical imaging”, Int. J. Mod. Phys. A 29, 1441003 (2014).

[68] Dekempeneer, Y. et al., "Targeted alpha therapy using short-lived alpha-particles and the promise of nanobodies as targeting vehicle", Expert Opin. Biol. Ther. 16, 1035 (2016).

[69] Reed, S. A. et al., "Efficient initiation of photonuclear reactions using quasimonoenergetic electron beams from laser wakefield acceleration", J. Appl. Phys. 102, 073103 (2007).

[70] Ledingham, K. W. D. et al., "High power laser production of short-lived isotopes for positron emission tomography", J. Phys. D: Appl. Phys. 37, 2341 (2004).

[71] Yang, X. et al., "Three beam acceleration from a laser-plasma wakefield accelerator and the energy apportioning question", Sci. Rep. 7, 43910 (2017).

[72] Klenke, A. et al., "Coherently combined 16-channel multicore fiber laser system”, Opt. Lett. 43, 1519 (2018).

[73] Zhang R. et al., "Single-mode millijoule fiber laser system with high pulse shaping ability", Optik 157, 1087 (2018).

[74] Mason, P. et al., "Kilowatt average power 100 J-level diode pumped solid state laser", Optica 4, 438 (2017).

[75] Budriunas, R et al., "53 W average power CEP-stabilized OPCPA system delivering $5.5 \mathrm{TW}$ few cycle pulses at $1 \mathrm{kHz}$ repetition rate", Opt. Express 25, 5797 (2017).

[76] Hattawy, M. et al., "First exclusive measurement of deeply virtual Compton scattering off He-4: Toward the 3D tomography of nuclei", Phys. Rev. Lett. 119, 202004 (2017).

[77] Jung, J. H., Choi, Y. and Chun Im, K., "PET/MRI: Technical challenges and recent advances", Nucl. Med. Mol. Imaging 50, 3 (2016).

[78] Zheng, X. et al., "Measurement of target and double-spin asymmetries for the $\vec{e} \vec{p} \rightarrow e \pi^{+}(n)$ reaction in the nucleon resonance region at low Q2", Phys. Rev. C 94, 045206 (2016).

[79] O’Donnell, D. et al., "First observation of excited states of Hg-173”, Phys. Rev. C 85, 054315 (2012).

[80] Scheck, M. et al., "Investigating the pygmy dipole resonance using beta decay", Phys. Rev. Lett. 116, 132501 (2016).

[81] Fleetwood, D. M. and Eisen, H. A., “Total-dose radiation hardness assurance”, IEEE Trans. Nucl. Sci. 50, 552 (2003). 